\title{
ON THE FORM OF THE FINITE-DIMENSIONAL PROJECTIVE REPRESENTATIONS OF AN INFINITE ABELIAN GROUP
}

\author{
N. B. BACKHOUSE
}

\begin{abstract}
If the locally compact abelian group $G$ has a finitedimensional unitary irreducible projective representation with factor system $\omega$ (i.e. $G$ has an $\omega$-rep), then a subgroup $G(\omega)$ is defined which fulfils three roles. First, the square-root of the index of $G(\omega)$ in $G$ is the dimension of every $\omega$-rep. Secondly, the $\omega$-reps of $G$ can be labelled by the dual group of $G(\omega)$, up to unitary equivalence. Thirdly, the essential projective form of an $\omega$-rep is determined by a unique projective representation of the finite group $G / G(\omega)$.
\end{abstract}

1. Introduction. In a recent paper [1], Bradley and the present author showed that, for a given factor system $\omega$, the finite-dimensional unitary irreducible projective representations of a locally compact abelian group $G$ could differ from one another essentially only by linear characters. Implicit in this statement is the result that these representations, denoted $\omega$-reps in [1], share a common dimension $d(\omega)$. It was shown further that, up to projective equivalence, all $\omega$-reps could be obtained by the induction of linear characters from a maximal $\omega$-symmetric subgroup $M(\omega)$-recall that a subgroup $H$ of $G$ is said to be $\omega$-symmetric if $\omega\left(h_{1}, h_{2}\right)=\omega\left(h_{2}, h_{1}\right)$ for all $h_{1}, h_{2} \in H$. However, $M(\omega)$ is not unique, so that this fact together with the rather elusive definition of the subgroup has prevented $M(\omega)$ from being a useful tool in the determination of $d(\omega)$ in any but the most trivial cases.

The main result of this paper provides among other things a further group theoretical characterisation of $d(\omega)$, which promises to make determinations of $d(\omega)$ more tractable than was the case previously. The new subgroup, $G(\omega)$, which is introduced for the above purpose, is further put to use, for it is shown that the members of a set of inequivalent $\omega$-reps of $G$ can be put into one-to-one correspondence with the members of the dual group of $G(\omega)$, and furthermore that the essential projective nature of an $\omega$-rep is that of a unique projective representation of the finite group $G / G(\omega)$.

Received by the editors June 19, 1972 and, in revised form, February 12, 1973.

AMS (MOS) subject classifications (1970). Primary 22D12, $20 \mathrm{C} 25$.

Key words and phrases. Locally compact abelian group, projective representation, factor system, $\omega$-regular class, $\omega$-symmetric subgroup.

(c) American Mathematical Society 1973 
First we recall a result of Frucht [2] on the finite group case, both for its future use and for the purpose of contrast of proof.

2. Finite abelian groups. It is known from the work of Schur [3], Kleppner [4], Conlon [5] and Harter [6] that the number, $n(\omega)$, of inequivalent $\omega$-reps of a finite group $G$ is equal to the number of $(\omega$-regular classes-an element $g \in G$ is said to be $\omega$-regular if $\omega(g, h)=\omega(h, g)$ for all $h$ in the centraliser of $g$. In the case that $G$ is abelian this reduces to the statement that $n(\omega)$ is equal to the number of elements $g \in G$ for which $c(g, h)=\omega(g, h) / \omega(h, g)$ is unity for all $h \in G$. Noting that $c$ is a bicharacter, for example see Kleppner [7], it follows simply that the $\omega$-regular elements of $G$ form a subgroup $G(\omega)$, whose order is $n(\omega)$. We recall two properties of $\omega$-reps. First, that the sum of the squares of the dimensions of a set of $n(\omega)$ inequivalent $\omega$-reps equals $|G|$. Secondly, that the $\omega$-reps of an abelian group share a common dimension $d(\omega)$. It follows at once that $d(\omega)^{2}=|G / G(\omega)|$. It is this result of Frucht [2] that we now show to be the case even for infinite abelian groups, when some of the above analysis becomes meaningless.

3. Infinite abelian groups. Suppose the locally compact abelian group $G$ has factor system $\omega$. According to Kleppner [4], it can be assumed that $\omega$ is normalised, that is $\omega\left(g, g^{-1}\right)=1$ for all $g \in G$. As above, if $G((1))$ is defined to be the set of all $\omega$-regular elements of $G$, then $G(\omega)$ has the group property and moreover is closed in the topological sense. The following two results are found to be useful in the statement and proof of the main result concerning the relationship between $G(\omega)$ and the (1)-dual of $G$.

Lemma 1. Let $G$ be a finite abelian group, and suppose $G(1)=\{e\}$. Then, up to unitary equivalence, $G$ has a unique $\left(\omega-\operatorname{rep} \pi\right.$, and $(\operatorname{dim} \pi)^{2}=|G|$.

Proof. This is a simple deduction from $\$ 2$ above. In fact a stronger result than Lemma 1 is possible. For it quickly follows from Theorems 3 and 4 of Kleppner [4] that if $G(\omega)=\{e\}$ and $G$ is discrete, then the hypothesis that $G$ is finite is equivalent to each of the hypotheses that $G$ has a type I $\omega$-dual or that $G$ has a unique irreducible $\omega$-representation.

Lemma 2. If $G$ is a locally compact abelian group possessing an (1)-rep, then $G(\omega)$ is of finite index in $G$.

Proof. We employ the subgroup $M(\omega)$, mentioned in $\S 1$ and studied in reference [1].

If $m \in M(\omega)$, then $g \rightarrow c(m, g)$ is a character of $G$ which takes the value one on $M(\omega)$. A priori, different choices of $m$ yield different characters. However, since $M(\omega)$ is of finite index in $G$, there are only a finite number 
of characters which are trivial on $M(\omega)$; indeed, by the Frobenius reciprocity theorem of Mackey [8], every such character must appear in the reduction of the induced representation $\chi_{0} \uparrow G$, where $\chi_{0}$ is the trivial character of $M(\omega)$. It follows that there exist elements $m_{i} \in M, i=1$, $2, \cdots, r \leqq d(\omega)$, such that for each $m \in M(\omega), g \rightarrow c(m, g)$ can be identified with $g \rightarrow c\left(m_{i}, g\right)$ for some $i$.

Now let $g_{j}, j=1,2, \cdots, d(\omega)$, be a set of coset representatives for $M(\omega)$ in $G$. If $g \in G$, then $g=g_{j} m$ for some $g_{j}$ and for some $m \in M$. Let $m$ be associated with the element $m_{i} \in M$ in the manner indicated above. Then by linearity, $c(g, h)=c\left(g_{j}, h\right) c(m, h)=c\left(g_{j}, h\right) c\left(m_{i}, h\right)=c\left(g_{j} m_{i}, h\right)$ for all $h \in G$. Again by linearity, and using the definition of $G(\omega)$, we see that $g^{-1} g_{j} m_{i} \in G(\omega)$. It follows that $G(\omega)$ is of index at most $d(\omega)^{2}$ in $G$. This concludes the proof.

We are now able to state and prove the main result.

THEOREM. Let $G$ be a locally compact abelian group, and suppose that $G(\omega)$ is of finite index, or equivalently that $G$ possesses an $\omega$-rep.

Then there exists a factor system $\Omega$ on $G / G(\omega)$ such that $(G / G(\omega))(\Omega)=$ $\{e\}$, and is such that $\omega$ is similar to the lift of $\Omega$. Assume without loss of generality that $\Omega$ lifts to $\omega$, and let $\pi$ be the unique $\Omega$-rep of $G / G(\omega)$. Then all the $\omega$-reps of $G$ are of the form $g \rightarrow \chi(g) \pi(\bar{g})$, where $\chi \in \hat{G}$ and $\bar{g}=G(\omega) g \in G / G(\omega)$, and all such representations are irreducible.

Finally $\chi \otimes \pi \cong \chi^{\prime} \otimes \pi$ if and only if $\chi, \chi^{\prime}$ coincide on $G(\omega)$.

Proof. Since $\omega$ is symmetric on $G(\omega)$, it is trivial there, so we may replace $\omega$ by a similar factor system which is identically one on $G(\omega)$. Supposing this is done, we apply Mackey's theory [9] to compute the $\omega$-reps of $G$ from those of $G(\omega)$. If $\theta$ is an $\omega$-rep of $G(\omega)$, which in this case means that $\theta$ is a character of $G(\omega)$, then it is soon checked that the little $\omega$-group (i.e. stability group) of $G$ at $\theta$ is $G$ itself. By Theorem 8.2 of [9] it follows that there exists a multiplier $\Omega$ on $G / G(\omega)$ and an extension $\theta^{\prime}$ of $\theta$ which satisfies $\theta^{\prime}\left(g_{1}\right) \theta^{\prime}\left(g_{2}\right)=\theta^{\prime}\left(g_{1} g_{2}\right) \omega\left(g_{1}, g_{2}\right) \Omega\left(\bar{g}_{1}, \bar{g}_{2}\right)^{-1}$, for all $g_{1}$, $g_{2} \in G$. In particular $\omega$ and the lift of $\Omega$ are similar, so that we may replace $\omega$ by the lift of $\Omega$. It also follows that $(G / G(\omega))(\Omega)=\{\bar{e}\}$.

If we extend $\theta$ to a character $\chi$ of $G$, then on the one hand $\chi$ is an ordinary representation, but on the other hand, according to the same theorem of Mackey [9], $\chi$ is a projective representation with factor system $\omega \tau$, where $\tau$ is lifted from $G / G(\omega)$. It follows that $\tau=\omega^{-1}$. Now we may conclude, again from [9], that the $\omega$-reps of $G$ are of the form $g \rightarrow \chi(g) \pi(\bar{g})$, where $\pi$ is the unique $\Omega$-rep of $G / G(\omega)$, and that $\chi_{1} \otimes \pi$ and $\chi_{2} \otimes \pi$ are equivalent if and only if $\chi_{1}, \chi_{2}$ agree on $G(\omega)$. This last statement asserts that there is a natural bijection between the dual of $G(\omega)$ and the $\omega$-dual of $G$. This concludes the proof. 
It is perhaps interesting to note that the $\omega$-reps of $G$ discussed above have easily computed characters (i.e. traces). Thus if $D=\chi \otimes \pi$, then $\operatorname{tr} D(g)=d(\omega) \chi(g)$ if $g \in G(\omega)$. However, $\operatorname{tr} D(g)=0$ if $g \notin G(\omega)$ for the following reason. Generally $\operatorname{tr} D(h)=c(g, h) \operatorname{tr} D(h)$ follows from the equation $D(g) D(h) D(g)^{-1}=c(g, h) D(h)$. But if $h \notin G(\omega)$ there exists at least one $g \in G$ for which $c(g, h) \neq 1$. This is hardly a surprising result, for already in the finite group case it is known from [3], [5] and [6] that the $\omega$-character of an $\omega$-rep is zero outside the $\omega$-regular classes, and indeed that $\omega$ can be so adjusted that the $\omega$-character is a class function.

4. Conclusion. We have seen in several ways the importance of the group $G(\omega)$ for the $\omega$-reps of an abelian group $G$. First, the size of $G(\omega)$ relative to $G$ gives the square of $d(\omega)$. Because the definition of $G(\omega)$ is rather more direct than that of the other relevant subgroup $M(\omega)$, it would seem that the new characterisation of $d(\omega)$ is of more value than that embodied in Theorem 3 of [1]. In practice the determination of the index $|G / G(\omega)|$ presents no great problem, and for example in the case of finitely generated groups the comparison of unit cell volumes, by the obvious determinantal generalisation of (6.21) of [10], provides a suitable method.

Secondly, we know that the $\omega$-reps of $G$ can be labelled by the characters of $G(\omega)$. To be computationally useful of course this result requires that the detailed structure of $\hat{G}(\omega)$ be known.

Thirdly, the burden of the projective nature of an $\omega$-rep has been put onto a unique projective representation of a finite abelian group, for whose further analysis the work of Schur [11] and Frucht [2] is available.

It is perhaps worth pointing out finally that special cases of the representations which we have been studying are quite familiar objects; indeed the present author [12] and Morris [13] have noted that much of the theory of generalised Dirac matrices by Morris [14], Barut [15] and Ramakrishnan, Raghavacharayulu [16] can be treated from the viewpoint of the projective representation theory of abelian groups. In fact the little $\omega$-group method rather quickly leads to the familiar tensor product expressions for the Dirac matrices which were originally obtained by Weyl and Brauer [17].

ACKNOWLEDGMENTS. The author wishes to thank the referee for his efforts to improve the appearance of the main result. Help in slimming down the proofs was also gratefully received from Miss Patricia Gard.

\section{BIBLIOGRAPHY}

1. N. B. Backhouse and C. J. Bradley, Projective representations of abelian groups, Proc. Amer. Math. Soc. 36 (1972), 260-266.

2. R. Frucht, Über die Darstellung endlicher Abelscher Gruppen durch Kollineationen, J. Math. 166 (1931), 16-29. 
3. I. Schur, Über die Darstellung der endlichen Gruppen durch gebrochene lineare Substitutionen, J. Math. 127 (1904), 20-50.

4. A. Kleppner, The structure of some induced representations, Duke Math. J. 29 (1962), 555-572. MR 25 \#5132.

5. S. B. Conlon, Twisted group algebras and their representations, J. Austral Math. Soc. 4 (1964), 152-173. MR 29 \#5921.

6. W. G. Harter, Algebraic theory of ray representations of finite groups, J. Mathematical Phys. 10 (1969), 739-752. MR 39 \#4296.

7. A. Kleppner, Multipliers on abelian groups, Math. Ann. 158 (1965), 11-34. MR 30 \#4856.

8. G. W. Mackey, On induced representations of groups, Amer. J. Math. 73 (1951), 576-592. MR 13, 106.

9. - Unitary representations of group extensions. I, Acta Math. 99 (1958), 265-311. MR 20 \#4789.

10. N. B. Backhouse and C. J. Bradley, Projective representations of space groups. I, Quart. J. Math. Oxford Ser. (2) 21 (1970), 203-222. MR 41 \#5510.

11. I. Schur, Untersuchungen über die Darstellung der endlichen Gruppe durch gebrochene lineare Substitutionen, J. Math. 132 (1907), 85-137.

12. N. B. Backhouse, The theory of projective representation: pure and applied, Senior Mathematics Prize Dissertation, Oxford, 1971 (unpublished).

13. A. O. Morris, Private communication.

14. —_, On a generalised Clifford algebra, Quart. J. Math. Oxford Ser. (2) 18 (1967), 7-12. MR 35 \#5461.

15. A. O. Barut, On the irreducible representations of a class of algebras and related projective representations, J. Mathematical Phys. 7 (1966), 1908-1910. MR 36 \#7385.

16. A. Ramakrishnan and I. V. V. Raghavacharayulu, $A$ note on the representations of Dirac groups, Sympos. on Theoretical Physics and Mathematics, vol. 8. Plenum, New York, 1968, pp. 25-32. MR 39 \#5100.

17. R. Brauer and H. Weyl, Spinors in n-dimensions, Amer. J. Math. 57 (1935), 425-449.

Department of Applied Mathematics, University of Liverpool, Liverpool, ENGLAND 